\title{
«Wir suchten als «Mavericks〉 stets von neuem den Rand der Herde»
}

Interview:

Bruno Kesseli

* Die Initialen HHB für Hans Heinrich Brunner sind im Schweizer Gesundheitswesen zu einer Art Markenzeichen geworden, weshalb sie im

Interview belassen werden.

Korrespondenz:

Dr. rer. pol. Heinz Locher

Postfach 266

CH-3000 Bern 15

heinz@locher-bern.ch

bkesseli@emh.ch
Für das Anfang dieses Jahres erschienene Buch «Die Schweiz hat das beste Gesundheitssystem - hat sie das wirklich?» haben zwei «Urgesteine» der gesundheitspolitischen Landschaft Schweiz als Co-Autoren zusammengefunden: Gesundheitsökonom Heinz Locher und der vor rund einem Jahr verstorbene frühere FMH-Präsident und BAG-Vizedirektor Hans Heinrich Brunner, zu dessen Vermächtnis das Werk geworden ist. Im Interview äussert sich Heinz Locher zum Buch.

"Die Schweiz hat das beste Gesundheitssystem - hat sie das wirklich?» ist als Gemeinschaftswerk zwischen Ihnen und dem kurz vor der Vollendung des Buchs leider verstorbenen Hans Heinrich Brunner entstanden. Wie kam es zu dieser Zusammenarbeit?

Heinz Locher: $\mathrm{HHB}^{*}$ und ich begegneten uns erstmals Anfang der 90er Jahre aus Anlass seines Projektes für einen neuen Arzttarif. Von da an haben sich unsere Wege in verschiedensten beruflichen Funktionen immer wieder gekreuzt, ohne dass wir aber je

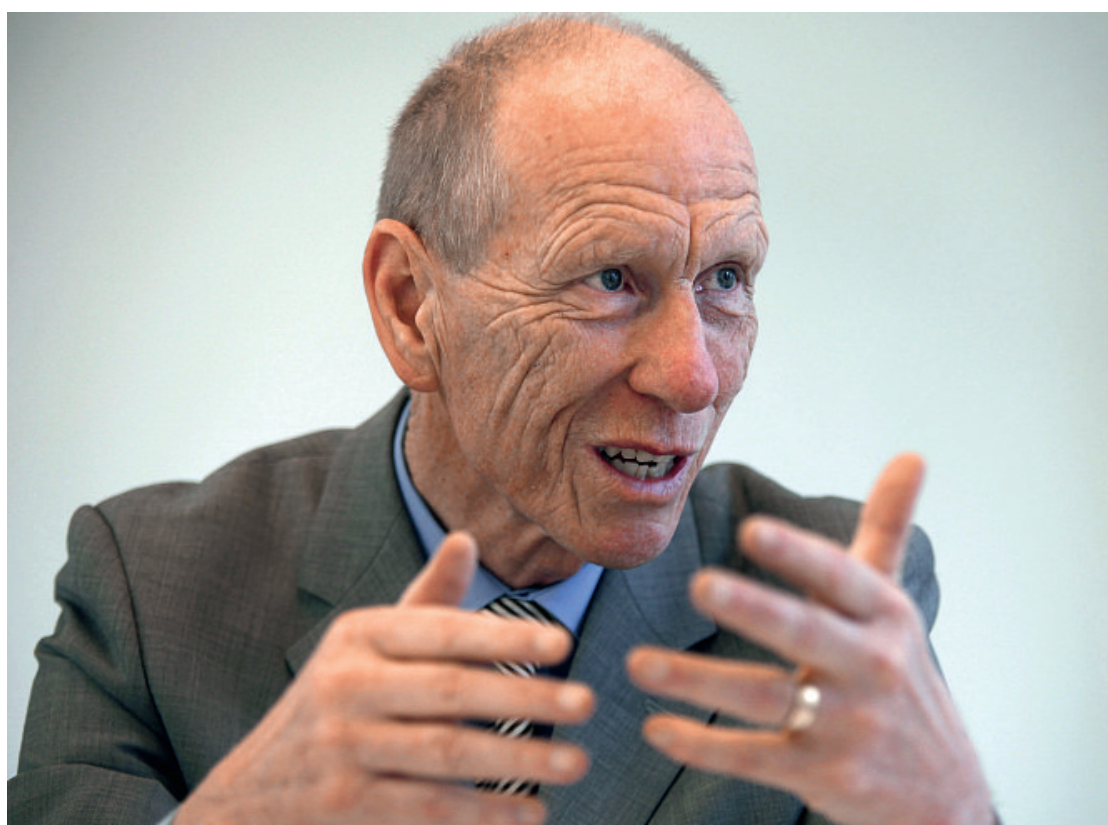

Versteht das Gesundheitssystem als entwicklungsfähiges, komplexes System, das nicht planbar ist: Heinz Locher. Foto: Beat Mathys. enger zusammengearbeitet hätten. Die von mir trotz aller biographischer Unterschiede verspürte Geistesverwandtschaft mit ihm entsprach offensichtlich auch seinem Eindruck von mir: Beide waren wir einerseits Entscheidungsträger in verantwortungsvollen Funktionen des Gesundheitswesens, blieben aber trotzdem immer auch «teilnehmende Beobachter», suchten als «Mavericks» stets von neuem den Rand der Herde und liessen uns nie voll vom «System» vereinnahmen. Als er mir im Mai 2008 vorschlug, wir könnten doch gemeinsam ein Buch über das schweizerische Gesundheitswesen schreiben, musste ich nicht lange überlegen und sagte spontan zu.

Welche Leitidee steht hinter dem Buch?

Die Grundidee ist ganz schlicht: Wir wollten mit einer praxisbezogenen, (selbst-)kritischen Schau einen Beitrag zur Weiterentwicklung unseres Gesundheitssystems leisten - ganz im Zeichen einer unbestechlichen kritischen Aufklärung, deren englische Bezeichnung mir besonders gut gefällt: «Enlightenment».

\section{«Wir wollten mit einer praxis- \\ bezogenen, (selbst-)kritischen Schau einen Beitrag zur Weiter- entwicklung unseres Gesundheits- systems leisten.»}

Sie bezeichnen Hans Heinrich Brunner als «Erstautor der meisten Kapitel» und schreiben dem Buch Vermächtnischarakter zu. Inwiefern ist diese Einstufung gerechtfertigt?

Entgegen der ursprünglichen Absicht, dass wir beide je etwa gleich viele Kapitel schreiben würden, entwickelte HHB eine eindrückliche Schreibdynamik, mit der ich schon rein zeitlich nicht mithalten konnte. Nachträglich schien es mir, er habe gespürt, dass seine Zeit engbegrenzt sei. Dieser Eindruck verstärkte sich anlässlich unserer Diskussionen und Gespräche, in welchen er mich durch die drängende Dringlichkeit seines Reformwillens beeindruckte. 


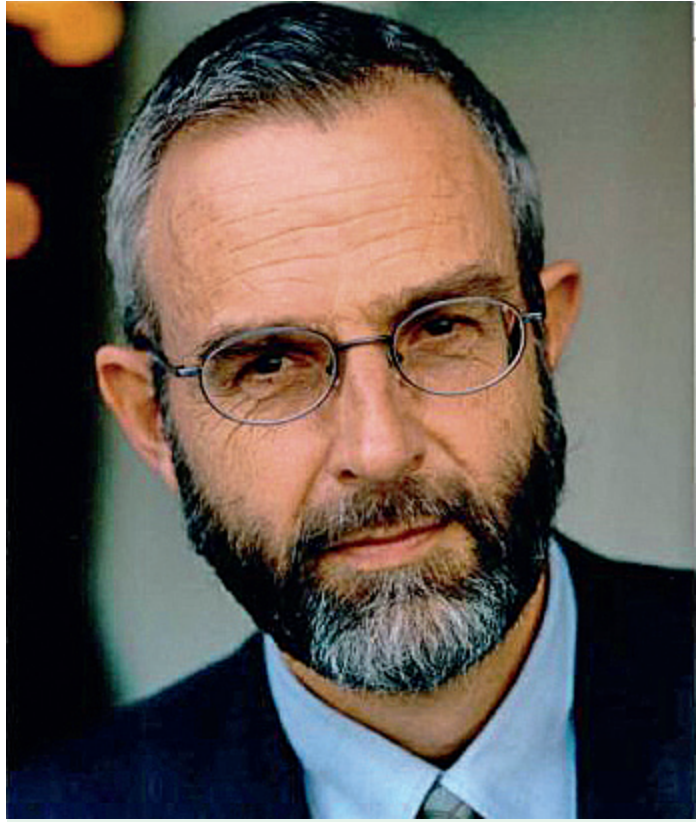

«Eindrückliche Schreibdynamik»: Hans Heinrich Brunner †

\section{«Verglichen mit einem Musikstück kann man sagen, dass die meisten Melodien von HHB stammen, das Arrangement hingegen von mir ist.»}

Nach welchen Kriterien nahmen Sie die Themenwahl vor, die gemäss Klappentext «weder den Hauptströmen noch den Mäandern der politischen Tagesdiskussion» folgt? Wenn ich mich vor Referaten selber vorstellen darf, bezeichne ich mich etwa als «bekennenden Ordoliberalen» und füge bei, das sei nicht identisch mit anarcholiberal. Der Staat soll Regeln aufstellen, deren Einhaltung überwachen und bei Fehlleistungen eingreifen, ohne aber selber handelnd tätig zu werden. Das Gesundheitssystem soll unter Beachtung seiner Eigenheiten gleichen Normen wie andere Wirtschaftsund Gesellschaftsbereiche unterstellt sein. Diese Grundhaltung hat nicht nur im von mir schon ganz früh redigierten Kapitel «Wettbewerb als Ordnungsprinzip» seinen Niederschlag gefunden, sondern wurde in praktisch allen Kapiteln zum Leitfaden. HHB hat seinerseits anlässlich seines kurz zurückliegenden Masterstudiums in Harvard viele Impulse empfangen, was Anlass zu Ausführungen über «Gesundheitswesen als System», «evidenzbasierte Medizin», «Varianz in der Medizin» usw. gab.

Obwohl das Buch mit etwas über 160 Seiten nicht sehr umfangreich ist, weist es thematisch eine beeindruckende Breite auf. Sind Schwerpunkte darunter, die Ihnen besonders am Herzen liegen?
Wir sind beide in den 1960er Jahren mit der damals neuen und aufregenden Kybernetik und Systemtheorie konfrontiert worden, eine «frühkindliche Prägung», die in unser ganzes Berufsleben gewirkt hat. Die beiden Kapitel «Ist ein Gesundheitssystem planbar?» und «Wettbewerb als Ordnungsprinzip» sind Ausdruck dieses Systemdenkens und des Denkens in Ordnungen. Sie bilden die beiden tragenden Pfeiler des Buches, die anderen Kapitel sind seine Brückenbogen.

Können Sie dies noch etwas ausführen?

Die sich aus den genannten Kapiteln ergebenden Schlussfolgerungen sind zentral: Wenn das Gesundheitswesen ein komplexes System mit vielfältigen iterativen Prozessen und Rückkoppelungen ist, entzieht es sich allen «Masterplänen» und «Gesamtkonzeptionen». Wer an zentraler Stelle entscheiden und handeln muss, braucht mehr Demut als Macherwillen, muss Systemzusammenhänge und mögliche Rückkoppelungen bestmöglich bedenken, nur schrittweise vorgehen, Zwischenergebnisse evaluieren. Anschaungsbeispiele, wie man nicht vorgehen darf, gibt es viele, es sei nur an die Revision des Analysetarifs erinnert.

Das «schönste», das heisst von mir als am feinsten empfundene Kapitel stammt übrigens aus der Feder von HHB und charakterisiert die Vorsteher des EDI zwischen 1987 bis 2010 von F. Cotti über R. Dreifuss zu P. Couchepin und D. Burkhalter.

Steckt nun im Buch mehr «Brunner» oder mehr «Locher»? Die Frage scheint nicht irrelevant, weil Sie - wie im Vorwort festgehalten - zunächst selbst vermuteten, dass «wir uns in wichtigen Fragen wohl nicht einig werden würden». Verglichen mit einem Musikstück kann man sagen, dass die meisten Melodien von HHB stammen, das Arrangement hingegen von mir ist.

In welchen Bereichen waren die Divergenzen zwischen Hans Heinrich Brunner und Ihnen am grössten, und wie haben Sie in diesen Fällen zu einer gemeinsam getragenen Aussage gefunden?

Es gab nur einen einzigen «heissen» Punkt: HHB hatte für sein Executive Master Studium in Harvard, das ihm viel bedeutete, die Fächer «Health Economics and Statistics» gewählt. Nun ist aber die in Harvard im Vordergrund stehende mathematisch dominierte Gesundheitsökonomie nicht gerade mein «cup of tea». Auch HHB griff sie und deren Menschenbild vor dem Hintergrund seiner ärztlichen Grundhaltung heftig an. Ich fand seinen Kampf gegen diesen Popanz nicht sehr relevant, bezeichneten sich doch die Urväter der von mir vertretenen Grundhaltung noch als Moralphilosophen. Sie waren also so ziemlich das Gegenteil der heutigen «Chicago Boys». Nach einigen Mailwechseln einigten wir uns darauf, dass diese Diskussion unsere Leserinnen und Leser wohl nicht besonders interessieren würde, und strichen die Passage. 
In der Analyse ist das Buch ziemlich schonungslos und greift auch heisse Eisen auf - man erhält durchaus den Eindruck, dass hier aus einer unabhängigen Position argumentiert wird. Was «Lösungsansätze» betrifft, kochen die Autoren aber auch nur mit Wasser. Könnten Sie sich mit dieser Bewertung anfreunden?

Ich habe unsere Reformvorschläge im Schlusskapitel HHB war leider schon verstorben - zusammengefasst und stehe nach wie vor voll und ganz zur Aussage, wonach das Gesundheitssystem als entwicklungsfähiges, komplexes System verstanden werden muss, das nicht planbar ist. Es muss durch permanente Evaluation des Geschehens als lernendes System ausgestaltet werden. Wenn wegen dieser Grundhaltung der Vorwurf formuliert wird, wir kochten auch nur mit Wasser, lässt mich das kalt, und ich gehe als «Maverick» an den Rand der Herde, die sich offenbar gerade zur Stampede sammelt, beispielsweise zur Lancierung der Einheitskasseninitiative.

Wenn Sie zwei Vorschläge aus Ihrem Buch zur Verbesserung unseres Gesundheitssystems realisieren könnten: Welche wären dies?

Ich bin schon mit einem Thema zufrieden: Meine Priorität heisst «Normalisierung des Gesundheitssystems», gleiche Behandlung wie alle anderen Wirtschafts- und Gesellschaftsbereiche. Also marktwirtschaftlich-wettbewerbliche Regelungen, Konsumentensouveränität, Ende der staatlichen Gängelung.

\section{«Meine Priorität heisst «Normalisierung des Gesund- heitssystems», gleiche Behandlung wie alle anderen Wirtschafts- und Gesellschaftsbereiche.»}

Das Buch ist ja schon einige Zeit im Handel und wurde mitunter - z.B. in der Handelszeitung - in einer Weise vorgestellt, die durchaus Zündstoff für Polemiken enthielt. Welche Reaktionen sind bisher zu Ihnen gedrungen?

Das auch von der Handelszeitung hervorgehobene Korruptionsthema hat offensichtlich viele angesprochen. Ich ziehe heute den weiter gefassten Begriff «fehlende Transparenz» vor. Hier fehlt es in vielen Bereichen des Gesundheitssystems noch an der dringend erforderlichen Sensibilisierung - Finanzierung

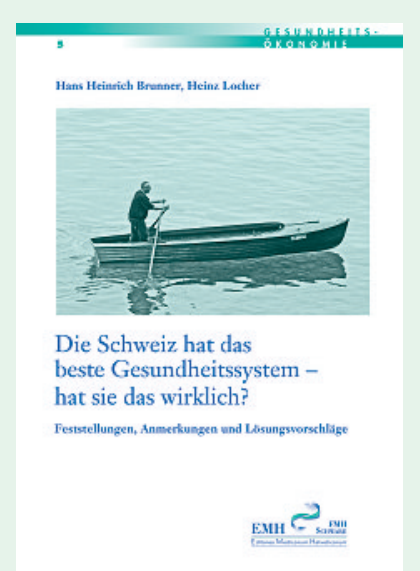

Hans Heinrich Brunner, Heinz Locher

Die Schweiz hat das beste Gesundheitssystem hat sie das wirklich?

Feststellungen, Anmerkungen und Lösungsvorschläge. Basel: EMH Schweizerischer Ärzteverlag; 2011.

165 Seiten. Broschiert. 35 CHF.

ISBN 978-3-03754-054-1

Für das Buch «Die Schweiz hat das beste Gesundheitssystem - hat sie das wirklich?» haben zwei profilierte Persönlichkeiten des schweizerischen Gesundheitswesens als Co-Autoren zusammengefunden. Hans Heinrich Brunner, der vor rund einem Jahr verstorbene frühere $\mathrm{FMH}$ Präsident und ehemalige Vizedirektor des Bundesamts für Gesundheit, wie auch der Berner Gesundheitsökonom Heinz Locher haben unser Gesundheitssystem wärend Jahrzehnten als Akteure und kritische Beobachter mitgeprägt. In ihrem Buch bieten die Autoren eine unabhängige Auseinandersetzung mit zentralen Aspekten des vermeintlich «besten Gesundheitssystems». Neben der kritischen und mitunter schonungslosen Analyse enthält das Werk auch zahlreiche Anregungen und Lösungsansätze.

von Weiterbildungsveranstaltungen, klinische Versuche, Gutachtertätigkeit usw.

Was müsste das Buch bewirken, damit Sie sich zufrieden zurücklehnen und sagen könnten: «Ziel erreicht?»

Der Weg ist das Ziel: permanente, kritisch reflektierte Weiterentwicklung. 